\title{
Visual Feedback Increases Postural Stability in Children with Autism Spectrum Disorder
}

Eszter Somogyi ${ }^{1}$, Eszter Kapitány ${ }^{1}$, Krisztina Kenyeres ${ }^{1}$, Nándor Donauer ${ }^{2}$, Jacqueline Fagard $^{3}$ and Anikó Kónya ${ }^{1}$

${ }^{1}$ Department of Cognitive Psychology, Eötvös Loránd University, Izabella u. 46, H-1064

Budapest, Hungary

${ }^{2}$ St. John Hospital - Buda Children's Hospital, Bolyai u. 5-9, H-1021, Budapest, Hungary

${ }^{3}$ Laboratoire Psychologie de la Perception, Université Paris Descartes, CNRS UMR 8158, Centre Biomédical des Saints-Pères, 75006 Paris, France

\section{Corresponding author}

Eszter Somogyi ${ }^{1}$ : esomogyi@gmail.com

Present address: Laboratoire Psychologie de la Perception, Université Paris Descartes, CNRS UMR 8158, Centre Biomédical des Saints-Pères, 75006 Paris, France

\section{Acknowledgements}

We would like to thank all the children and their families for their participation and interest in this study. We would also like to thank Dr. Szabó Csilla and Mohos Katalin (Eötvös Loránd University, Bárczi Gusztáv Faculty of Special Education) for conducting the participants’ non-verbal IQ and receptive language assessment.

The last author was supported by a grant nr K-81641 of the National Scientific Research Foundation of Hungary. 
Visual Feedback Increases Postural Stability in Children with Autism Spectrum Disorder

\section{Highlights}

- We examined postural control and ability to improve posture in children with ASD.

- ASD children were significantly more unstable than TD controls at baseline.

- They improved significantly when given visual feedback of their center of pressure.

- Posture training with visual feedback might improve general motor control in ASD. 
Visual Feedback Increases Postural Stability in Children with Autism Spectrum Disorder

\section{1. Introduction}

2 The beneficial effect of training on the motor functioning of children with autism spectrum

3 disorders (ASD) is well documented (Lang et al., 2010; Sowa \& Meulenbroek, 2012). Still,

4 the mechanisms that underlie this effect are rarely targeted by empirical research. In the

5 current study, we wished to capture ability of children with ASD to use visual cues for improving their postural control, an important component of gross motor development.

1.1. Autism spectrum disorders and motor function

From the first clinical descriptions of ASD, poor motor skills have been commonly reported (Kanner, 1943). Empirical studies confirm that children with ASD experience both gross and fine motor delays and show atypical motor patterns (Ghaziuddin \& Butler, 1998; Green et al., 2009; Ming, Brimacombe, \& Wagner, 2007; Miyahara et al., 1997; Provost, Lopez, \& Heimerl, 2007, for reviews see: Gidley-Larson \& Mostofsky, 2006; Gowen \& Hamilton, 2013). Motor function depends greatly on postural control, the fundamental and earlydeveloping ability to maintain equilibrium by keeping or returning the center of body mass over its base of support (Horak, 1987). This was shown for instance in a sub-analysis conducted by Whyatt and Craig (2012) of the motor performance of children with ASD on the Movement Assessment Battery for Children (Henderson \& Sugden, 1992), which assesses manual dexterity, ball skills and balance. They found that the motor skill deficits indicated by this test were specifically apparent in activities demanding core balance ability, such as static balance and catching a ball. A recent study (Mache \& Todd, 2016) directly comparing motor

21 skills and postural control in children with ASD has confirmed that a significant predictor of fundamental motor skill performance (locomotion and ball skills) in ASD is postural control. 
Indeed, studies that have assessed postural stability in ASD by measuring balance time have generally found difficulties sustaining a posture for longer periods of time (Ghaziuddin, Butler, Tsai, \& Ghaziuddin, 1994; Green et al., 2009; Jansiewicz et al., 2006; Noterdaeme, Mildenberger, Minow, \& Amorosa, 2002; Papadopoulos et al., 2012, though see: Weimer, Schatz, Lincoln, Ballantyne, \& Trauner, 2001 for diverging results). Research that used force plates to record the exact amount of movement made by participants when trying to hold a posture have also consistently reported increased sway in children with ASD during quiet stance (Fournier et al., 2010; Gepner \& Mestre, 2002; Kohen-Raz, Volkmar, \& Cohen, 1992; Memari et al., 2013; Minshew, Sung, Jones, \& Furman, 2004, though see: Molloy, Dietrich, \& Bhattacharya, 2003 for opposite results).

Balance is regulated through the afferent signals from the somatosensory, the vestibular and the visual systems (Peterka \& Benolken, 1995). Experiments that manipulated afferent inputs show abnormal compensatory functioning between the three subsystems in ASD. For example, in Weimer et al.’s study (2001), while children and young adults with Asperger Syndrome (AS) balanced on one leg with eyes open for a similar amount of time as controls, they balanced for significantly less time when standing on one foot with eyes closed. Similarly, Molloy et al. (2003) found that when their vision was occluded, children with ASD had significantly more difficulties in maintaining balance than controls, whether or not somatosensory input was also modified, which suggests an overreliance on visual cues. Two recent studies have further confirmed this visual dependency by showing that children with ASD show more postural sway than controls when their eyes are closed (Stins, Emck, de Vries, Doop, \& Beek, 2015) or while performing a visual searching task as compared to sway during an auditory digit span task (Memari, Ghanouni, Shayestehfar, Ziaee, \& Moshayedi, 2014).

Minshew et al. (2004) compared how individuals with ASD (children and adults) and controls 
compensate for disrupted visual, vestibular or somatosensory inputs and found the relative importance of the latter to be the greatest. In this study, the postural stability of individuals with ASD was significantly reduced compared to controls when somatosensory input was disrupted alone or in combination with the disruption of the visual input. The authors also revealed a specific developmental trajectory for postural stability in persons with ASD.

Postural control did not begin to improve until the age of 12 years in children with ASD and never achieved adult levels, whereas in controls, it improved steadily from 5 to 15-20 years, before it plateaued.

An alternative hypothesis put forward by Gepner et al. (1995; 2002) is that atypical postural function in ASD does not derive from basic motor impairments but from a deficit in visualmotion integration, which can be captured in reduced reactivity to fast moving visual stimulation. They reported that children with ASD were posturally hyporeactive to visually perceived environmental motion in comparison with typically developing (TD) controls (Gepner et al., 1995). Greffou et al. (2012) further explored the question by assessing postural response in fully immersive dynamic virtual tunnels. Similarly to Gepner et al. (1995; 2002), they also found abnormal postural reactivity in participants with ASD, but only in the younger group (aged 12-15 years) and for specific oscillation frequencies.

Although the role of postural reactivity remains uncertain, the above studies underscore the relative importance of visual cues for maintaining balance in ASD.

\subsection{The effect of IQ}

Postural stability seems to be linked to IQ (Minshew et al., 2004) and level of functioning in ASD (Gepner \& Mestre, 2002; Kohen-Raz et al., 1992; Memari et al., 2013). Children with ASD who have intellectual disability are more likely to show reduced postural stability even in static conditions with a stable floor and normal visual input (Kohen-Raz et al., 1992; Memari et al., 2013; Minshew et al., 2004). Cognitively able children with ASD on the other 
hand seem to catch up with TD children from the age of about 12 years, after which abnormal functioning has been found only for challenging conditions where afferent inputs were modified (Greffou et al., 2012; Minshew et al., 2004; Weimer et al., 2001). Only few studies, however, have explored postural skills in children with ASD below the age of 12, with some confirming prolonged delay until this age (Fournier et al., 2010; Memari et al., 2013;

Minshew et al., 2004), but not others (Molloy et al., 2003; Price, Shiffrar, \& Kerns, 2012).

Inconsistent findings may be due to the variability of assessment methods and sway measures as well as to samples often covering a wide age range.

\subsection{The present study}

Our present study had two aims. First, we wished to disambiguate existing data on the postural skills of children with ASD below the age of 12 by measuring postural stability in children with ASD without intellectual disability aged 5-11 years. We hypothesized that examining a large sample and a close age range with precise posturography, we would find reduced baseline postural stability in this young population (Fournier et al., 2010; Memari et al., 2013; Minshew et al., 2004).

Secondly, we wished to explore the effect of visual feedback on postural performance. Our second hypothesis was that, given their strong reliance on visual cues when maintaining balance (Gepner et al., 1995; Gepner \& Mestre, 2002; Greffou et al., 2012; Memari et al., 2014; Molloy et al., 2003), children with ASD would improve in their postural performance if provided with contingent visual feedback of the movements of their center of pressure (CoP).

\section{Methodology}

\subsection{Participants}

We recruited 18 children with ASD (14 boys) from two schools for children with ASD in Budapest, Hungary. Each child had completed the assessment procedure required for a formal diagnosis of ASD in order to enter the schools. During this procedure children were examined 
by a multidisciplinary team composed of a general practitioner, a clinical psychiatrist and an educational psychologist. They were diagnosed with autistic disorder according to DSM-IVTR (American Psychiatric Association, 2000) criteria. The Autism Diagnostic Observation Schedule-Generic (ADOS-G; Lord et al., 2000) and the Autism Diagnostic Interview-Revised (ADI-R; Lord et al. 1994), were used to establish diagnoses. These were administered by the educational psychologist, who was qualified for using these diagnostic tools. The schools’ professionals assessed the severity of the children's ASD symptoms with the Childhood Autism Rating Scale (CARS; Schopler, Reichler, \& Renner, 1993) upon admission and each schoolyear. All the children with ASD participating in the study had CARS scores between 30 and 37 (mean 34,5 points) indicating mild to moderate autism (Mayes et al., 2012). We excluded children who had any genetic/medical conditions commonly comorbid with ASD (Fragile X-, Down- or Tourette syndrome, seizures, epilepsy), physical impairments or handicaps by screening the children's medical history. None of the children were under medication during the testing period.

Their ages ranged from 5 to 11 years (65 to 133 months, mean: 94 months). All the children had non-verbal IQs within the average range and average receptive language levels, as measured with Raven’s Colored Progressive Matrices (R-CPM, Raven, 1993, Rózsa, 2006) and the Peabody Picture Vocabulary Test (PPVT-R, Dunn, 1997), respectively. Receptive language level was measured in order to ascertain that children with ASD would understand the task instructions. Parents were asked to fill the Movement Assessment Battery for Children - 2 Checklist (MABC -2 Checklist, Henderson, Sugden \& Barnett, 2007), which focuses on how a child manages everyday situations in school or at home and indicates whether a child is likely to have gross motor abnormalities. According to this measure none of the children had gross motor impairments.

As the control group, we recruited 12 healthy age-matched TD children (8 boys) from a 
mainstream public school in Budapest, Hungary.

125 Their ages ranged from 7 to 9 years (86 to 112 months, mean: 97 months). Their non-verbal

126 IQs, as measured with Raven’s Colored Progressive Matrices (R-CPM, Raven, 1993, Rózsa,

127 2006) were found to be within the average range. TD children were also screened with the

128 MABC -2 Checklist (Henderson, Sugden \& Barnett, 2007), which indicated that none of them

129 had gross motor impairments. We assumed that healthy, TD children attending a regular public

130 school would understand the simple instructions of our task, their receptive vocabulary level

131 was therefore not measured. Exclusion criteria were known genetic, mental or neurological

132 disorders or physical impairments, which were screened with a further parent questionnaire.

133 None of the parents reported the presence of any such conditions.

134 Written consent to recruit and test in the schools was first obtained from each school's

135 principal. We distributed information letters briefly describing the study via the school to

136 parents of children between 5 to 11 years of age. Tear-off forms were appended to the letter,

137 allowing us to contact parents who were interested in the study in order to provide further

138 information and to obtain their signed informed consent. The study was approved by the

139 Medical Ethics committee of the University of Budapest.

140 Participants' descriptive statistics are reported in Table 1. The two groups of children with

141 ASD and TD children were well matched on chronological age $(\mathrm{t}(28)=0.43, \mathrm{p}=.33)$.

142 Regarding mental age, the TD group had significantly higher IQs ( $(28)=4.91, \mathrm{p}<.0001)$.

143 However, as all participants had IQs within the average range and were above clinical criteria

144 for impaired IQ (with IQ scores above 70), the groups were retained. For more precise

145 analyses, the group of children with ASD was split into two subgroups based on IQ; children

146 with ASD - IQ>100 ( $\mathrm{n=10)}$ and children with ASD - IQ 80-100 (n=8).

148 Please insert Table 1 about here. 
151 Postural sway measurements were performed using the Virtual Human Interface platform ${ }^{\odot}$

152 (Digital Elite/PanoCAST, Inc., Los Angeles, CA), which employs real-time graphics and

153 imaging to provide various visual (or auditory) stimuli related to specific rehabilitative needs.

154 The hardware consisted of a HP Probook laptop communicating via Bluetooth connectivity

155 with a Nintendo Wii balance board (511 x 316 x $53.2 \mathrm{~mm}$ ) that registered the actual location 156 and movement of the CoP of the participants' body. The Wii balance board has been found to

157 be a reliable and valid tool to measure balance in research and clinical settings (Clark et al.,

158 2010). Data generated by the balance were processed by custom software named Cyber Care

159 Clinic $^{\odot}$ (Digital Elite/PanoCAST, Inc., Los Angeles, CA), which transposed CoP movements

160 to the laptop’s 17-inch monitor (resolution of 42 pixels per $\mathrm{cm}$ ). The child’s CoP was

161 represented by a blue rectangle $(1.6 \mathrm{~cm} \mathrm{x} 1 \mathrm{~cm})$ that moved in conjunction with the movements

162 of the child's CoP within a greater white circle. Figure 1 shows the visual feedback presented

163 to children on the monitor.

164

165 Please insert Figure 1 about here.

166

\subsection{Measurements}

168

Cyber Care Clinic $^{\circledR}$ software calculated two postural sway measures: (1) participants’ Sway

169 Area (SA), the area of the outer envelope created by the x-y plot of the movement pattern of

170 the participants' CoP, and (2) Sway Length (SL), the total distance traversed by the CoP.

171 Cyber Care Clinic ${ }^{\odot}$ performed these calculations with consideration of the weight and the

172 height of each participant. As CoP movements were transposed to a [-1,1] normalized space,

173 measurements were relative, non-dimensional values with no units. 
175 The experiments took place within the schools in a quiet room that was familiar to the child

176 (such as the school library). Experimenters were therefore not blind to children's group membership. One experimenter managed the software while the other communicated with the child. Both ensured that the child understood the task and stood correctly with arms next to

179 his or her body, heels touching and eyes on the monitor. Throughout the session, verbal

180 instructions were simple and standardized in order to minimize any confounding elements of 181 language and comprehension.

182 During the tasks (except the Baseline Condition) the balance board was placed on the floor $1830.8 \mathrm{~m}$ from the table on which the computer was located. The monitor's center was at the eye184 level of the child. Each session consisted of the following three phases, 60s long each.

185 1. Baseline Condition: the child was asked to stand still on the balance board during 60s, without performing any movement. The child could not see the monitor. In order to obtain steady state results, the first and last 5 seconds were removed from the data and only the remaining 50 seconds were analyzed.

2. Training: 'Move the blue box on the screen' game. The experimenter asked the child to stand on the balance board, this time facing the monitor. She then showed to the child a small blue square on the monitor and explained that he or she could move this 'blue box' by swaying his or her body. The blue square moved in conjunction with the movements of the child's CoP. The aim of this 60 s familiarization period was to train children to use the apparatus and to ensure that they understood that the movement of their CoP was represented on the screen. Data recorded during familiarization was not analyzed.

3. Visual Feedback Condition: 'Keep the blue box still' game. The child remained on the 198 balance board, was asked to stand comfortably and to keep as still as possible so that 
the 'blue box' would not move. The trial lasted 60s, during which we recorded children's postural performance. As in the Baseline Condition, only 50 seconds of the data were analyzed.

2.5. Statistical analysis

Analyses were conducted on the average of data recorded during each phase. Mean SA and

SL across Conditions (Baseline vs. Visual Feedback) in children with ASD and TD children were compared. All statistical tests were performed using SPSS software version 17 (SPSS Inc., Chicago, IL, USA). The level of significance was set at $\mathrm{p}<0.05$.

\section{Results}

Figure 2 shows examples for scatter plots generated by the postural performance of a child with ASD and a TD child in the Baseline and the Visual Feedback Conditions. A mixeddesign ANOVA with Condition (Baseline or Visual Feedback) as within-subjects factor and Diagnosis (ASD or TD) as between-subjects factor revealed significant effects of Visual Feedback on both SA $\left(F(1,28)=9.48, p=.005, \eta_{p}^{2}=.253\right)$ and $\operatorname{SL}(F(1,28)=573, p<.0001$, $\left.\eta_{\mathrm{p}}{ }^{2}=.953\right)$. We found interactions between Condition and Diagnosis $(\mathrm{F}(1,28)=4.51, \mathrm{p}=.043$,

$214 \eta_{\mathrm{p}}{ }^{2}=.139$ for SA and $\mathrm{F}(1,28)=22.94, \mathrm{p}<.0001, \eta_{\mathrm{p}}{ }^{2}=.45$ for SL), suggesting that contingent 215 visual feedback of CoP had a greater effect on postural control in children with ASD than in 216 TD children.

217 Figure 3 shows mean SA and SL of children with ASD and TD children as a function of 218 Condition. Subsequent comparisons of means are presented below.

220 Please insert Figures 2 and 3 about here.

223 In the Baseline Condition postural stability was significantly lower in children with ASD than 
224 in TD children for both SA $(\mathrm{t}(28)=3.13, \mathrm{p}<.01)$ and $\mathrm{SL}(\mathrm{t}(28)=4.36, \mathrm{p}<.0001)$ measures.

225 Table 2 shows comparisons of mean baseline SA and SL for the two subgroups of children 226 with ASD, determined by level of IQ. We found that SA was significantly greater in both the 227 children with ASD - IQ>100 subgroup $(n=10 ; t(20)=3.08, p<.01)$ and the children with 228 ASD - IQ 80-100 subgroup $(\mathrm{n}=8 ; \mathrm{t}(18)=4.08, \mathrm{p}<.001)$ than in TD children. Similarly, 229 baseline SL was significantly greater in both the children with ASD - IQ>100 subgroup $230(\mathrm{n}=10 ; \mathrm{t}(20)=3.08, \mathrm{p}<.01)$ and the children with ASD - IQ 80-100 subgroup $(\mathrm{n}=8 ; \mathrm{t}(18)=$ 4.08, $\mathrm{p}<.001$ ) than in controls. Our first hypothesis was thus confirmed, as baseline SA and SL were greater in children with ASD than in TD children, independently of IQ.

234 Comparisons of mean SA and SL of children with ASD in the Baseline and the Visual 235 Feedback Conditions revealed that postural stability increased when visual feedback was provided, as both SA $(\mathrm{t}(17)=2.4, \mathrm{p}<.05)$ and $\mathrm{SL}(\mathrm{t}(17)=3.31, \mathrm{p}<.01)$ decreased significantly (see Figure 3). These results confirmed our second hypothesis; the postural performance of children with ASD improved when contingent visual feedback was provided 239 of the movements of their CoP.

240 Although they improved remarkably, children with ASD still had a significantly greater SA $(\mathrm{t}(28)=2.83, \mathrm{p}<.01)$ and SL $(\mathrm{t}(28)=2.83, \mathrm{p}<.01)$ than TD children. In the TD group, no difference in SA or SL was found; their postural stability was comparable to baseline in the Visual Feedback Condition.

244 We again compared means for the two subgroups of children with ASD separately (see Table 245 2). Just like the greater group, children with ASD in the IQ 80-100 subgroup ( $\mathrm{n}=8$ ) improved 246 significantly in their postural stability when provided visual feedback of the movement of 247 their $\operatorname{CoP}(\mathrm{Z}=-2.09, \mathrm{p}=.037)$. However, even their improved SA remained significantly 248 larger than that of TD children $(\mathrm{t}(18)=2.02, \mathrm{p}<.05)$. Children with ASD in the IQ $>100$ 
subgroup ( $\mathrm{n}=10)$ also improved in their postural stability when provided visual feedback, but

250 the difference between their SA in the Baseline and the Visual Feedback Conditions did not reach significance. Just as in the greater group though, their improved SA was still significantly larger than that of TD children $(\mathrm{t}(20)=3.95, \mathrm{p}<.001)$. These comparisons show that the effect of visual feedback was greater in the group of children with ASD with slightly

254 lower IQ.

\section{Discussion}

Children with ASD often show atypical motor patterns (Gidley-Larson \& Mostofsky, 2006;

Gowen \& Hamilton, 2013), which might in part be due to an immature postural control (Mache \& Todd, 2016; Whyatt \& Craig, 2012). Firstly, our findings confirm the presence of this deficit in childhood by showing that postural stability is reduced below 12 years of age in children with ASD, even during quiet stance (Fournier et al., 2010; Memari et al., 2013;

Minshew et al., 2004). Secondly, we provide new insight into postural instability by showing that it can be improved in a specific, facilitating environment, which in our case consisted of providing contingent visual feedback of the child's CoP movements. Thirdly, we found that postural instability was linked to IQ. Although children with ASD in our study were all above 265 clinical criteria for impaired IQ (with IQ scores above 70), similarly to earlier data (Minshew et al., 2004), we observed that children with ASD who had an IQ between 80 and 100 produced greater SAs than children with ASD with an IQ above 100. Interestingly, although

268 both groups improved, children in the lower IQ group benefited more from visual feedback 269 and reached greater stability than children in the higher IQ group.

270 It has been proposed that the common neural substrate linking postural and motor deficits in 271 ASD could be the cerebellum (Nayate, Bradshaw, \& Rinehart, 2005), which optimizes motor 272 performance in a given context and supports initial motor skill learning. Structural and 273 functional abnormalities of the cerebellum in ASD have been reported by numerous studies 
274 (for a review, see: D'Mello \& Stoodley, 2015), supporting the cerebellar hypothesis of these

275 disorders (e.g.: Courchesne, Yeung-Courchesne, Press, Hesselink, \& Jernigan, 1988; see

276 Fatemi et al., 2012 for a review). Still, as multiple regions of the brain show abnormalities in

277 this complex syndrome, further studies are required to clarify to what extent ASD can be

278 considered a disorder of the cerebellum.

279 The first limitation of our study is the modest sample size, which allowed analyses on the effect 280 of IQ only on small subgroups of children with ASD, all above the clinical criteria of intellectual 281 disability. Thus, the beneficial effect of visual feedback (and its selectivity) needs to be 282 confirmed by investigating the effect of postural training in children with ASD who have 283 intellectual disability. Also, possible comorbid symptoms of ADHD could not be ruled out 284 within this sample, as the DSM-IV-TR (APA, 2000) precludes a diagnosis of ADHD if ASD is 285 present.

286 Secondly, in the absence of comparison data from children with other developmental disorders, 287 the individual contributions ASD and developmental disorder per se remain unclear at present. 288 Deficits in postural control have in fact been associated with other developmental disorders 289 such as attention deficit hyperactivity disorder, Tourette syndrome, developmental coordination 290 disorder, cerebral palsy, and hearing loss (for a review, see; Memari, Ghanouni, Shayestehfar, 291 \& Ghaheri, 2014).

292 Thirdly, we would like to note that the MABC -2 Checklist (Henderson, Sugden \& Barnett, 293 2007) we used to assess gross motor functioning in our samples is a relatively coarse-grained 294 measure that may not detect dysfunctions in the sub-clinical domain. With this tool we only 295 wished to exclude gross motor problems that could have interfered with balance performance, 296 it did not allow for us to explore correlation between motor skills and postural control. A 297 recent study however (Mache \& Todd, 2016) that used more precise measures of fundamental 298 motor skill performance has confirmed correlation between the two, showing that a significant 
predictor of fundamental motor skill performance (locomotion and ball skills) in ASD is

300 postural control.

301 We conclude that in a specialized setting adapted to their needs, in our case their preference

302 for relying on real-time visual cues, children with ASD can learn to correct their posture. In

303 practice we suggest that using similar postural or motor tasks with a Wii balance board for

304 instance could well complement early interventions for CWA. Lang and colleagues (2010)

305 conducted a systematic review of studies focusing on the effects of physical exercise in

306 individuals with ASD and found that following motor interventions stereotypy, aggression and

307 off-task behaviors decrease. Similarly, balance training early in development may help not

308 only to improve motor abilities, but also to alleviate ASD symptoms.

\section{Acknowledgements}

We would like to thank all the children and their families for their participation and interest in this study.

We are immensely grateful to Takács Barnabás for developing the custom software named Cyber Care Clinic $^{\odot}$ (Digital Elite/PanoCAST, Inc., Los Angeles, CA, USA) and for assisting with the analyses of the data.

We would also like to show our gratitude to Dr. Szabó Csilla and Mohos Katalin (Eötvös Loránd University, Bárczi Gusztáv Faculty of Special Education) for conducting the participants’ IQ and receptive language assessment.

The last author was supported by a grant nr K-81641 of the National Scientific Research Foundation of Hungary.

\section{References}


American Psychiatric Association. (2000). Diagnostic and Statistical Manual of Mental Disorders (4th ed., text rev.). Washington DC.

Clark, R. A., Bryant, A. L., Pua, Y., McCrory, P., Bennell, K., \& Hunt, M. (2010). Validity and reliability of the Nintendo Wii Balance Board for assessment of standing balance. Gait Posture, 31(3), 307-310. doi: 10.1016/j.gaitpost.2009.11.012

Courchesne, E., Yeung-Courchesne, R., Press, G. A., Hesselink, J. R., \& Jernigan, T. L. (1988). Hypoplasia of cerebellar vermal lobules VI and VII in autism. $N$ Engl J Med, 318(21), 1349-1354. doi:10.1056/nejm198805263182102

D'Mello, A. M., \& Stoodley, C. J. (2015). Cerebro-cerebellar circuits in autism spectrum disorder. Frontiers in Neuroscience, 9. doi: 10.3389/fnins.2015.00408

Dunn, L. M. (1997). Peabody Picture Vocabulary Test (Vol. Third Edition). Minneapolis: American Guidance Service.

Fatemi, S. H., Aldinger, K. A., Ashwood, P., Bauman, M. L., Blaha, C. D., Blatt, G. J., . . . Welsh, J. P. (2012). Consensus paper: pathological role of the cerebellum in autism. Cerebellum, 11(3), 777-807. doi:10.1007/s12311-012-0355-9

Fournier, K. A., Kimberg, C. I., Radonovich, K. J., Tillman, M. D., Chow, J. W., Lewis, M. H., .. . Hass, C. J. (2010). Decreased static and dynamic postural control in children with autism spectrum disorders. Gait Posture, 32(1), 6-9. doi: 10.1016/j.gaitpost.2010.02.007

Gepner, B., Mestre, D., Masson, G., \& de Schonen, S. (1995). Postural effects of motion vision in young autistic children. Neuroreport, 6(8), 1211-1214.

Gepner, B., \& Mestre, D. R. (2002). Brief report: postural reactivity to fast visual motion differentiates autistic from children with Asperger syndrome. J Autism Dev Disord, 32(3), 231-238.

Ghaziuddin, M., \& Butler, E. (1998). Clumsiness in autism and Asperger syndrome: a further report. J Intellect Disabil Res, 42 ( Pt 1), 43-48.

Ghaziuddin, M., Butler, E., Tsai, L., \& Ghaziuddin, N. (1994). Is clumsiness a marker for Asperger syndrome? Journal of Intellectual Disability Research, 38(5), 519-527.

Gidley-Larson, J. C., \& Mostofsky, S. H. (2006). Motor Deficits in Autism. In R. Tuchman \& I. Rapin (Eds.), Autism: A neurological disorder of early brain development. (pp. 231247). London NW3 5RN England: Mac Keith Press.

Gowen, E., \& Hamilton, A. (2013). Motor abilities in autism: a review using a computational context. J Autism Dev Disord, 43(2), 323-344. doi: 10.1007/s10803-012-1574-0

Green, D., Charman, T., Pickles, A., Chandler, S., Loucas, T., Simonoff, E., \& Baird, G. (2009). Impairment in movement skills of children with autistic spectrum disorders. Dev Med Child Neurol, 51(4), 311-316. doi: 10.1111/j.1469-8749.2008.03242.x

Greffou, S., Bertone, A., Hahler, E. M., Hanssens, J. M., Mottron, L., \& Faubert, J. (2012). Postural hypo-reactivity in autism is contingent on development and visual environment: a fully immersive virtual reality study. J Autism Dev Disord, 42(6), 961970. doi: 10.1007/s10803-011-1326-6

Henderson, S.E. \& Sugden, D.A.. (1992). Movement Assessment Battery for Children.

Henderson S.E., Sugden D.A., Barnett A.L. (2007) Movement Assessment Battery for Children - 2 Examiner's Manual. London: Harcourt Assessment.

Horak, F. B. (1987). Clinical measurement of postural control in adults. Phys Ther, 67(12), 1881-1885.

Jansiewicz, E. M., Goldberg, M. C., Newschaffer, C. J., Denckla, M. B., Landa, R., \& Mostofsky, S. H. (2006). Motor signs distinguish children with high functioning autism and Asperger's syndrome from controls. J Autism Dev Disord, 36(5), 613-621. doi: 10.1007/s10803-006-0109-y

Kanner, L. (1943). Autistic disturbances of affective contact. Nervous Child, 2, 217-250. 
Kohen-Raz, R., Volkmar, F. R., \& Cohen, D. J. (1992). Postural control in children with autism. J Autism Dev Disord, 22(3), 419-432.

Lang, R., Koegel, L. K., Ashbaugh, K., Regester, A., Ence, W., \& Smith, W. (2010). Physical exercise and individuals with autism spectrum disorders: A systematic review.

Research in Autism Spectrum Disorders, 4(4), 565-576. doi: http://dx.doi.org/10.1016/j.rasd.2010.01.006

Mache, M. A., \& Todd, T. A. (2016). Gross motor skills are related to postural stability and age in children with autism spectrum disorder. Research in Autism Spectrum Disorders, 23, 179-187. doi: http://dx.doi.org/10.1016/j.rasd.2016.01.001

Mayes, S. D., Calhoun, S. L., Murray, M. J., Morrow, J. D., Yurich, K. K. L., Cothren, S., . . . Petersen, C. (2012). Use of the Childhood Autism Rating Scale (CARS) for Children With High Functioning Autism or Asperger Syndrome. Focus on Autism and Other Developmental Disabilities, 27(1), 31-38. doi: 10.1177/1088357611406902

Memari, A. H., Ghanouni, P., Gharibzadeh, S., Eghlidi, J., Ziaee, V., \& Moshayedi, P. (2013). Postural sway patterns in children with autism spectrum disorder compared with typically developing children. Research in Autism Spectrum Disorders, 7(2), 325-332. doi: http://dx.doi.org/10.1016/j.rasd.2012.09.010

Memari, A. H., Ghanouni, P., Shayestehfar, M., Ziaee, V., \& Moshayedi, P. (2014). Effects of visual search vs. auditory tasks on postural control in children with autism spectrum disorder. Gait Posture, 39(1), 229-234. doi: http://dx.doi.org/10.1016/j.gaitpost.2013.07.012

Ming, X., Brimacombe, M., \& Wagner, G. C. (2007). Prevalence of motor impairment in autism spectrum disorders. Brain Dev, 29(9), 565-570. doi: 10.1016/j.braindev.2007.03.002

Minshew, N. J., Sung, K., Jones, B. L., \& Furman, J. M. (2004). Underdevelopment of the postural control system in autism. Neurology, 63(11), 2056-2061.

Miyahara, M., Tsujii, M., Hori, M., Nakanishi, K., Kageyama, H., \& Sugiyama, T. (1997). Brief report: motor incoordination in children with Asperger syndrome and learning disabilities. J Autism Dev Disord, 27(5), 595-603.

Molloy, C. A., Dietrich, K. N., \& Bhattacharya, A. (2003). Postural stability in children with autism spectrum disorder. J Autism Dev Disord, 33(6), 643-652.

Nayate, A., Bradshaw, J. L., \& Rinehart, N. J. (2005). Autism and Asperger's disorder: Are they movement disorders involving the cerebellum and/or basal ganglia? Brain Research Bulletin, 67(4), 327-334. doi: http://dx.doi.org/10.1016/j.brainresbull.2005.07.011

Noterdaeme, M., Mildenberger, K., Minow, F., \& Amorosa, H. (2002). Evaluation of neuromotor deficits in children with autism and children with a specific speech and language disorder. Eur Child Adolesc Psychiatry, 11(5), 219-225. doi: 10.1007/s00787-002-0285-z

Papadopoulos, N., McGinley, J., Tonge, B., Bradshaw, J., Saunders, K., Murphy, A., \& Rinehart, N. (2012). Motor proficiency and emotional/behavioural disturbance in autism and Asperger's disorder: another piece of the neurological puzzle? Autism, 16(6), 627-640. doi: 10.1177/1362361311418692

Peterka, R. J., \& Benolken, M. S. (1995). Role of somatosensory and vestibular cues in attenuating visually induced human postural sway. Exp Brain Res, 105(1), 101-110.

Price, K. J., Shiffrar, M., \& Kerns, K. A. (2012). Movement perception and movement production in Asperger's Syndrome. Research in Autism Spectrum Disorders, 6(1), 391-398. doi: http://dx.doi.org/10.1016/j.rasd.2011.06.013

Provost, B., Lopez, B. R., \& Heimerl, S. (2007). A comparison of motor delays in young children: autism spectrum disorder, developmental delay, and developmental concerns. 
J Autism Dev Disord, 37(2), 321-328. doi: 10.1007/s10803-006-0170-6

Raven, J. (1993). Coloured progressive matrices-sets $a, a b, b$. Oxford: Oxford Psychologists.

Schopler, E., Reichler, R. J., \& Renner, B. R. (1993). The Childhood Autism Rating Scale (CARS). Los Angeles: Western Psychological Services.

Sowa, M., \& Meulenbroek, R. (2012). Effects of physical exercise on Autism Spectrum Disorders: A meta-analysis. Research in Autism Spectrum Disorders, 6(1), 46-57. doi: http://dx.doi.org/10.1016/j.rasd.2011.09.001

Stins, J. F., Emck, C., de Vries, E. M., Doop, S., \& Beek, P. J. (2015). Attentional and sensory contributions to postural sway in children with autism spectrum disorder. Gait Posture, 42(2), 199-203. doi: 10.1016/j.gaitpost.2015.05.010

Weimer, A. K., Schatz, A. M., Lincoln, A., Ballantyne, A. O., \& Trauner, D. A. (2001a). "Motor" impairment in Asperger syndrome: evidence for a deficit in proprioception. $J$ Dev Behav Pediatr, 22(2), 92-101.

Whyatt, C. P., \& Craig, C. M. (2012). Motor skills in children aged 7-10 years, diagnosed with autism spectrum disorder. J Autism Dev Disord, 42(9), 1799-1809. doi: 10.1007/s10803-011-1421-8. 


\section{Figures}

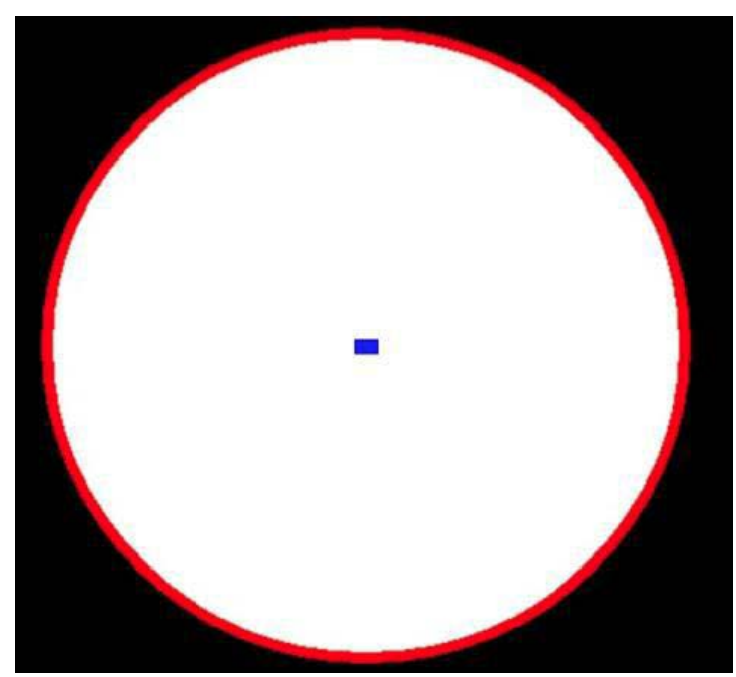

Figure 1 Visual feedback presented to children on the computer monitor during the 'Move the blue box' familiarization game and the 'Keep the blue box still' postural task. The blue square moved contingently with the movements of children's center of pressure.

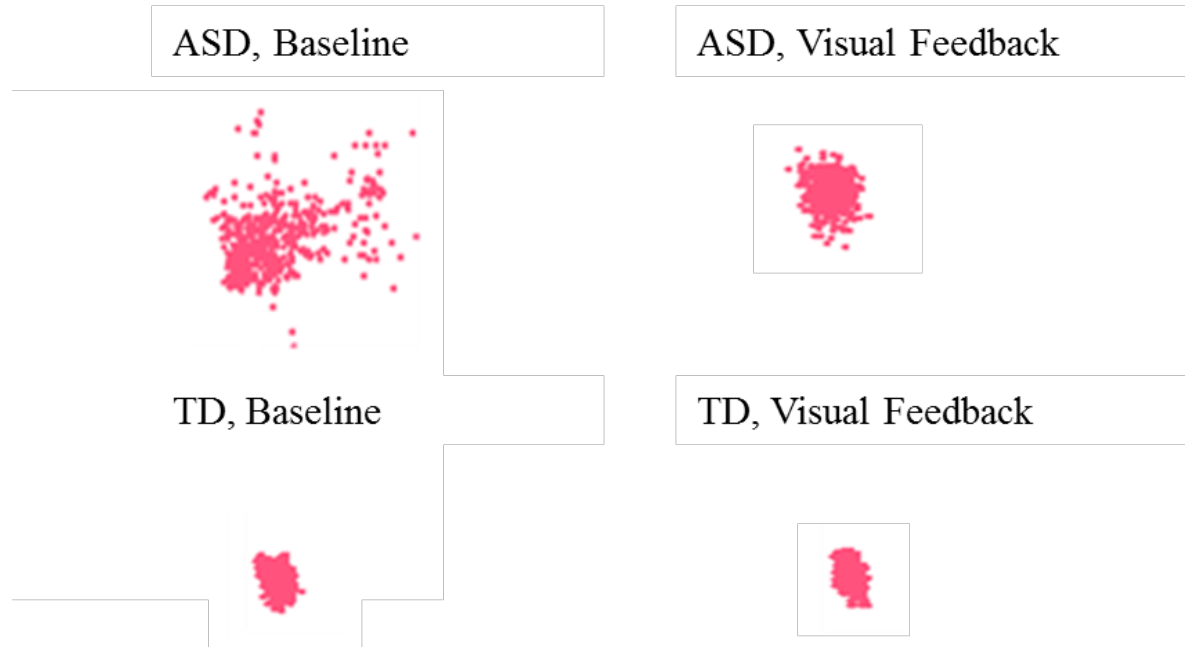

Figure 2 Scatter plots showing the movement of two 8-year-old subjects' center of pressure (CoP). The two plots on the top belong to a child with autism spectrum disorder (ASD), whereas the two on the bottom belong to a typically developing (TD) child. The plots on the left show the pattern of CoP movement when the child was standing quietly with eyes open on a firm surface in the Baseline Condition. Those on the right show pattern of sway in the Visual Feedback Condition. We can see that the sway area (SA) of the child with ASD is larger in the Baseline Condition than the SA of the TD child. This area shrinks close to normal when visual feedback is provided. 


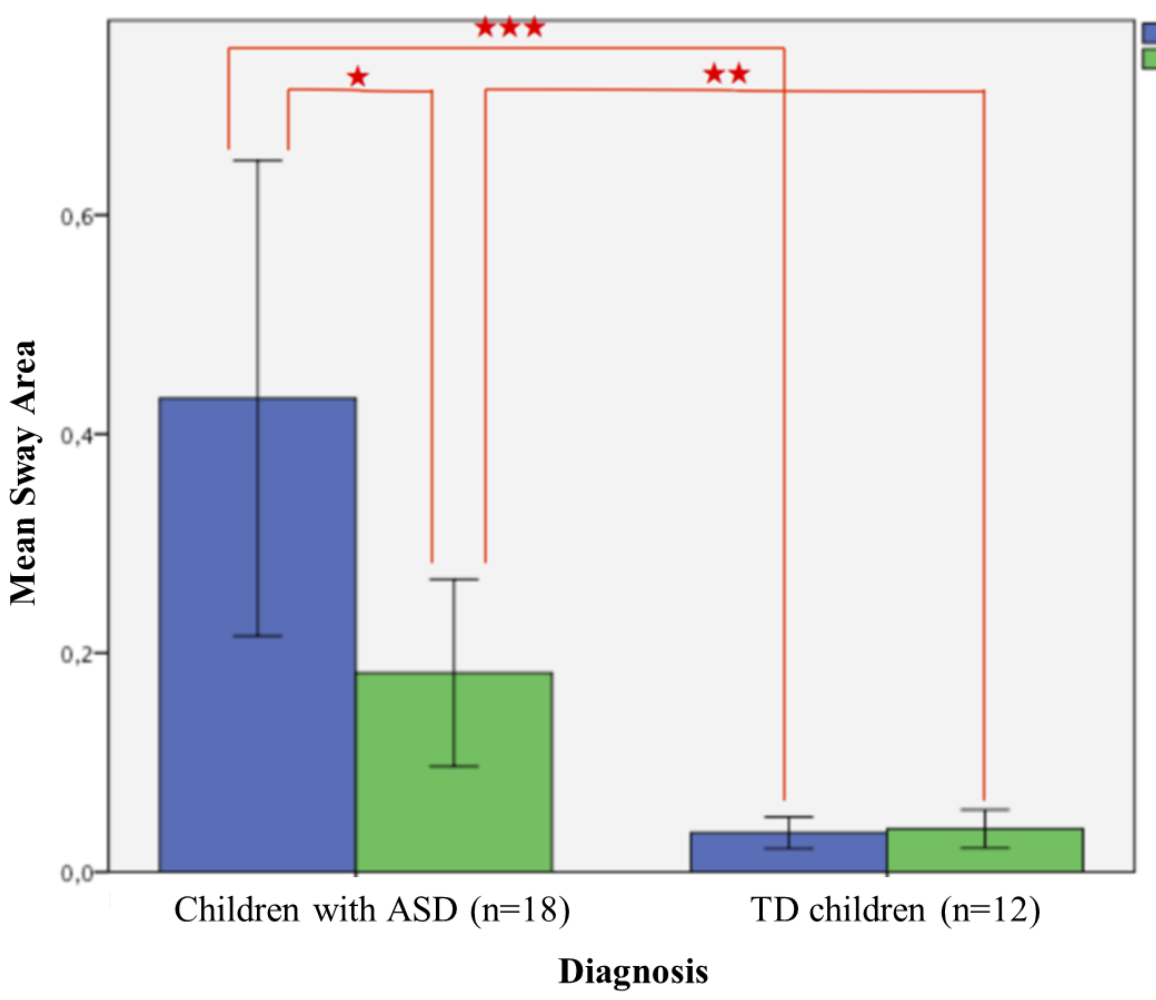

Baseline Condition

Visual Feedback Condition

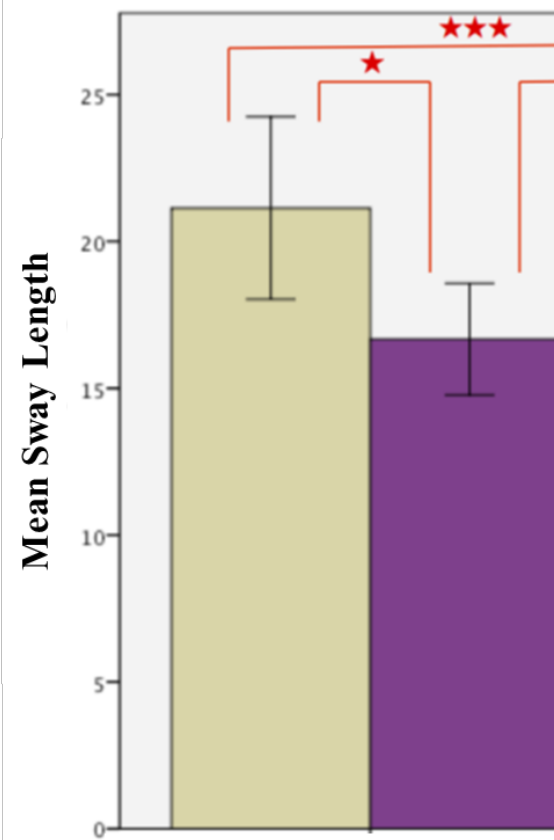

Baseline Condition

Visual Feedback Condition

Children with ASD $(\mathrm{n}=18)$

TD children $(\mathrm{n}=12)$

\section{Diagnosis}

Figure 3 Children's postural sway measured in Mean Sway Area and Sway Length as a function of Diagnosis (children with autism spectrum disorder (ASD) vs. typically developing (TD) children) and Condition (Baseline vs. Visual Feedback). A single asterisk indicates significance at $\mathrm{p}<.05$, two asterisks indicate $\mathrm{p}<.01$ and three indicate $\mathrm{p}<.001$. 
Visual Feedback Increases Postural Stability in Children with Autism Spectrum Disorder

\section{Tables}

\begin{tabular}{lllllll} 
& \multicolumn{2}{l}{ ASD (n=18) } & & \multicolumn{2}{l}{ TD (n=12) } \\
\cline { 2 - 6 } & $\mathrm{M}$ & Std.dev. & & $\mathrm{M}$ & Std.dev. & $\mathrm{p}$ \\
\hline Age (months) & 94 & 18.5 & & 96 & 9.6 & n.s. \\
Non-verbal IQ (R-CPM) & 98 & 17.9 & & 124 & 7.3 & $<.0001$ \\
Receptive language (PPVT-R) & 81 & 20 & NA & NA & NA \\
ASD symptom severity (CARS) & 35 & 2.23 & NA & NA & NA
\end{tabular}

Table 1 Descriptive statistics of the two groups of participants: children with autism spectrum disorder (ASD) and typically developing (TD) children. Non-verbal IQ was measured with Raven's Colored Progressive Matrices (R-CPM) in both groups. For children with ASD receptive language level was assessed with the Peabody Picture Vocabulary Test (PPVT-R) and symptom severity with the Childhood Autism Rating Scale (CARS). CARS scores indicate mild to moderate autism in our sample of children with ASD.

\begin{tabular}{|c|c|c|c|c|c|c|c|}
\hline & \multicolumn{3}{|c|}{ Baseline Condition } & \multicolumn{3}{|c|}{$\underline{\text { Visual Feedback Condition }}$} & \multirow[b]{2}{*}{ TD } \\
\hline & & ASD IQ $>100(n=10)$ & ASD IQ $80-100(n=8)$ & TD & ASD IQ $>100(n=10)$ & ASD IQ $80-100(n=8)$ & \\
\hline \multirow{2}{*}{ Sway Area } & Mean & $0.3282^{\star}$ & $0.5159 \star \star \star \star ⿱ 一 ⿻ 上 丨$ & 0.0358 & 0.2344 & $0.1397^{\star}$ & 0.0394 \\
\hline & SD & 0.2503 & 0.5419 & 0.0227 & 0.6637 & 0.1600 & 0.0275 \\
\hline \multirow{2}{*}{ Sway Length } & Mean & $21.37^{\star}$ & 20.95 & 12.87 & 16.82 & 16.56 & 12.00 \\
\hline & SD & 0.66 & 8.57 & 2.35 & 0.4065 & 5.23 & 1.83 \\
\hline
\end{tabular}

Table 2 Postural sway parameters as a function of Condition in two subgroups of children with autism spectrum disorder (ASD) determined by level of IQ, compared with typically developing (TD) children. Asterisks in the Baseline Condition column indicate significant differences between means as compared to the TD group. Asterisks in the Visual Feedback Condition column indicate significant differences between means as compared to the Baseline Condition. A single asterisk indicates significance at $\mathrm{p}<.05$, two asterisks indicate $\mathrm{p}<.01$. 